Helsinki University of Technology Institute of Mathematics Research Reports

\title{
GOAL-ORIENTED A POSTERIORI ERROR ESTIMATES FOR TRANSPORT PROBLEMS
}

Dmitri Kuzmin Sergey Korotov 

Helsinki University of Technology Institute of Mathematics Research Reports

\section{GOAL-ORIENTED A POSTERIORI ERROR ESTIMATES FOR TRANSPORT PROBLEMS}

Dmitri Kuzmin Sergey Korotov 
Dmitri Kuzmin, Sergey Korotov: Goal-oriented a posteriori error estimates for transport problems; Helsinki University of Technology Institute of Mathematics Research Reports A563 (2009).

\begin{abstract}
Some aspects of goal-oriented a posteriori error estimation are addressed in the context of steady convection-diffusion equations. The difference between the exact and approximate values of a linear target functional is expressed in terms of integrals that depend on the solutions to the primal and dual problems. Gradient averaging techniques are employed to separate the element residual and diffusive flux errors without introducing jump terms. The dual solution is computed numerically and interpolated using higher-order basis functions. A node-based approach to localization of global errors in the quantities of interest is pursued. A possible violation of Galerkin orthogonality is taken into account. Numerical experiments are performed for centered and upwind-biased approximations of a $1 D$ boundary value problem.
\end{abstract}

AMS subject classifications: $65 \mathrm{~N} 15,65 \mathrm{~N} 50,76 \mathrm{M} 30$

Keywords: stationary convection-diffusion equations, the finite element method, a posteriori error estimates, goal-oriented quantities, mesh adaptation

\title{
Correspondence
}

Institute of Applied Mathematics (LS III)

Dortmund University of Technology

Vogelpothsweg 87

D-44227 Dortmund

Germany

Institute of Mathematics

Helsinki University of Technology

P.O. Box 1100

FI-02015 TKK

Finland

kuzmin@math.uni-dortmund.de, sergey.korotov@hut.fi

ISBN 978-951-22-9758-0 (print)

ISBN 978-951-22-9759-7 (PDF)

ISSN 0784-3143 (print)

ISSN 1797-5867 (PDF)

Helsinki University of Technology

Faculty of Information and Natural Sciences

Department of Mathematics and Systems Analysis

P.O. Box 1100, FI-02015 TKK, Finland

email: math@tkk.fi http://math.tkk.fi/ 


\section{Introduction}

Numerical simulation of transport phenomena (convection and/or diffusion) plays an increasingly important role in science and engineering. The accuracy and reliability of computational methods depends on the choice of discretization techniques and, to a large extent, on the quality of the underlying mesh. Nowadays, adaptive mesh refinement techniques are widely used to reduce discretization errors in a computationally efficient way. Sometimes the location of critical zones, such as boundary and interior layers, is known. However, in most cases, mesh adaptation is an iterative process which involves estimation of numerical errors by means of certain a posteriori feedback mechanisms.

The derivation of a posteriori error estimates is aimed at obtaining computable lower and/or upper bounds for certain quantities of interest. In the case of convection-dominated transport problems, the global energy norm ceases to be a good measure of the numerical error. One of the most promising current trends in Computational Fluid Dynamics is goal-oriented adaptivity, whereby the duality argument is employed to derive an estimate for the magnitude of a given target/output functional $[1,6,7,16,17,19]$. The most prominent representative of such error estimators is the Dual-WeightedResidual (DWR) method of Becker and Rannacher [3, 4]. Remarkably, it is applicable not only to self-adjoint elliptic PDEs but also to hyperbolic conservation laws $[9,10]$.

In most cases, Galerkin orthogonality is involved in the derivation of goal-oriented a posteriori estimates by the DWR method. For the numerical solution to possess this property, the discretization must be performed by the Galerkin finite element method, and the resulting algebraic equations must be solved exactly. These requirements are rarely satisfied in practice due to numerical integration, round-off errors, and slack tolerances for iterative solvers. Last but not least, various stabilization terms or flux/slope limiters may be responsible for a (local) loss of Galerkin orthogonality. As a result, an extra term needs to be included in the error estimate for the DWR method. This part is computable but its localization, i.e., distribution among individual elements/nodes is a nontrivial task. Existing localization procedures [2] exploit the nature of the underlying discretization and are not universally applicable.

In the present paper, we address goal-oriented error estimation for stationary transport equations. The methodology to be presented is completely independent of the numerical scheme used to compute the approximate solution. The underlying localization procedure differs from that for the classical DWR method in a number of respects. First, integration by parts is applied to an averaged gradient so as to avoid the arising of jump terms at interelement boundaries. In the context of pure diffusion problems, gradient averaging has already been used in goal-oriented estimates [11, 12, 15] but the approach to be presented is more general and based on different premises. Second, the error in the quantity of interest is expressed in terms of nodal 
values [18], which yields a nonoscillatory distribution of weighted residuals. Moreover, errors due to the lack of Galerkin orthogonality are localized in a simple and natural way. The conversion of nodal errors to element contributions is straightforward.

The derivation of the above error estimate is followed by a discussion of algorithmic details and application to a one-dimensional convection-diffusion problem. The availability of analytical solutions makes it possible to perform a detailed analysis of accuracy and to identify the major sources of error.

\section{Goal-oriented error estimation}

Consider the Dirichlet problem that models steady convection and diffusion of a conserved scalar quantity $u(\mathbf{x})$ in a domain $\Omega$ with boundary $\Gamma$

$$
\left\{\begin{aligned}
\nabla \cdot(\mathbf{v} u-\varepsilon \nabla u)=f & & \text { in } \Omega, \\
u=b & & \text { on } \Gamma
\end{aligned}\right.
$$

where $\mathbf{v}(\mathbf{x})$ is a known velocity field, $\varepsilon>0$ is a constant diffusion coefficient, $f(\mathbf{x})$ is a volumetric source/sink, and $b(\mathbf{x})$ is the prescribed boundary data.

A variational form of problem (1) can be constructed by the weighted residual method using integration by parts. Let $H^{1}(\Omega)$ be the Sobolev space of square integrable functions with first derivatives in $L^{2}(\Omega)$. Furthermore, let $H_{0}^{1}(\Omega)$ denote a subspace of functions from $H^{1}(\Omega)$ vanishing on the boundary $\Gamma$. The problem statement becomes: Find $u \in H^{1}(\Omega)$ such that $u=b$ on $\Gamma$ and

$$
a(w, u)=(w, f), \quad \forall w \in H_{0}^{1}(\Omega),
$$

where the bilinear form $a(\cdot, \cdot)$ and the $L^{2}$ scalar product $(\cdot, \cdot)$ are defined by

$$
\begin{aligned}
& a(w, u)=\int_{\Omega} w \nabla \cdot(\mathbf{v} u) \mathrm{d} \mathbf{x}+\int_{\Omega} \nabla w \cdot(\varepsilon \nabla u) \mathrm{d} \mathbf{x}, \quad \forall w, u \in H^{1}(\Omega) \\
& (w, f)=\int_{\Omega} w f \mathrm{~d} \mathbf{x}, \quad \forall w, f \in L^{2}(\Omega)
\end{aligned}
$$

Let $u_{h} \in H^{1}(\Omega)$ be a numerical solution of problem (2) satisfying the Dirichlet boundary condition $u_{h}=b$ on $\Gamma$. It is convenient to define $u_{h}$ as a finite element interpolant of nodal values computed by an arbitrary numerical scheme.

Numerical errors can be quantified using the residual of the weak form (2)

$$
\rho\left(w, u_{h}\right)=(w, f)-a\left(w, u_{h}\right), \quad \forall w \in H_{0}^{1}(\Omega) .
$$

Note that the value of $\rho\left(w, u_{h}\right)$ depends on the choice of $w$. This weight should carry information about the propagation of errors and goals of simulation.

In many cases, the quantities of interest vary linearly with the solution. For example, if the solution behavior in a subdomain $\omega \subset \Omega$ is of particular 
interest, then a possible definition of the linear target functional $j(\cdot)$ reads $[11,12]$

$$
j(u)=\int_{\omega} u \mathrm{~d} \mathbf{x}, \quad \forall u \in L^{2}(\omega) .
$$

In order to estimate the value of $j(u)=j\left(u_{h}\right)+j(e)$ and/or the error $j(e)$ in the quantity of interest for the primal problem (2), consider the associated dual or adjoint problem $[3,4]$ which reads: Find $z \in H_{0}^{1}(\Omega)$ such that

$$
a(z, w)=j(w), \quad \forall w \in H_{0}^{1}(\Omega)
$$

Hence, the error $j\left(u-u_{h}\right)$ and residual (5) are related by the formula

$$
j\left(u-u_{h}\right)=a\left(z, u-u_{h}\right)=\rho\left(z, u_{h}\right) .
$$

An arbitrary approximation $z_{h} \in H_{0}^{1}(\Omega)$ to the exact solution $z$ of the dual problem $(7)$ can be used to decompose the so-defined error as follows

$$
j\left(u-u_{h}\right)=\rho\left(z-z_{h}, u_{h}\right)+\rho\left(z_{h}, u_{h}\right) .
$$

The value of $\rho\left(z-z_{h}, u_{h}\right)$ depends on the unknown solution $z$ of the dual problem, whereas the contribution of $\rho\left(z_{h}, u_{h}\right)$ is computable. If Galerkin orthogonality holds for the pair of approximations $u_{h}$ and $z_{h}$, then $\rho\left(z_{h}, u_{h}\right)=$ 0 .

The error representation (9) leads to a posteriori error estimates of the form

$$
\left|j\left(u-u_{h}\right)\right| \leq \Phi\left(z_{h}, u_{h}\right)+\Psi\left(z_{h}, u_{h}\right),
$$

where $\Phi\left(z_{h}, u_{h}\right)$ and $\Psi\left(z_{h}, u_{h}\right)$ represent the upper bounds for the magnitudes of the residuals $\rho\left(z-z_{h}, u_{h}\right)$ and $\rho\left(z_{h}, u_{h}\right)$, respectively. Let $\Phi_{i}$ and $\Psi_{i}$ be the local bounds associated with nodes (vertices) of the mesh such that

$$
\Phi\left(z_{h}, u_{h}\right)=\sum_{i} \Phi_{i}, \quad \Psi\left(z_{h}, u_{h}\right)=\sum_{i} \Psi_{i}
$$

The corresponding element contributions $\eta_{k}$ to (10) are supposed to satisfy

$$
\Phi\left(z_{h}, u_{h}\right)+\Psi\left(z_{h}, u_{h}\right)=\eta\left(z_{h}, u_{h}\right)=\sum_{k} \eta_{k} .
$$

The derivation of (10)-(12) is nontrivial since (i) the dual solution $z$ is generally unknown and (ii) the decomposition of the global error into nodal/element contributions is nonunique. In what follows, we elaborate on the approximation of $z$ and present a practical approach to estimation of local errors.

\section{Approximation of dual solutions}

Since the exact dual solution $z$ is usually unknown, it needs to be replaced by a suitable approximation $\hat{z} \approx z$. By virtue of (9), the setting $\hat{z}:=z_{h}$ yields 
the estimate $j\left(u-u_{h}\right) \approx \rho\left(z_{h}, u_{h}\right)$ which is useless if $\rho\left(z_{h}, u_{h}\right)=0$ due to Galerkin orthogonality. If $z_{h}$ belongs to the same finite-dimensional space as $u_{h}$, then $\hat{z}$ should reside in a different subspace of $H^{1}(\Omega)$ and possess higher accuracy.

For simplicity, we assume that the nodal values of the approximate solutions $u_{h}$ and $z_{h}$ are defined on the same mesh. Consider the finite element interpolants

$$
u_{h}=\sum_{j} u_{j} \varphi_{j}, \quad z_{h}=\sum_{i} z_{i} \varphi_{i}, \quad \hat{z}=\sum_{i} z_{i} \psi_{i}
$$

where the piecewise-polynomial basis functions $\varphi_{i}$ and $\psi_{i}$ correspond, e.g., to the $P_{1} / P_{2}$ or $Q_{1} / Q_{2}$ approximation on a pair of embedded meshes with spacing $h$ and $2 h$, respectively. For details, we refer to Schmich and Vexler [18].

Alternatively, the space spanned by $\left\{\varphi_{i}\right\}$ may be enriched by adding quadratic bubble functions [15]. In this case, some postprocessing of $z_{h}$ or solution of local subproblems is required to determine the additional degrees of freedom.

\section{Residuals and diffusive flux errors}

Given $\hat{z} \approx z$, the first term in the right-hand side of $(9)$ is approximated by

$$
\begin{aligned}
\rho\left(\hat{z}-z_{h}, u_{h}\right) & =\int_{\Omega}\left(\hat{z}-z_{h}\right)\left(f-\nabla \cdot\left(\mathbf{v} u_{h}\right)\right) \mathrm{d} \mathbf{x} \\
& -\varepsilon \int_{\Omega} \nabla\left(\hat{z}-z_{h}\right) \cdot \nabla u_{h} \mathrm{~d} \mathbf{x} .
\end{aligned}
$$

In the classical DWR method, elementwise integration by parts is applied to the second integral. Due to the discontinuity of the diffusive flux, this leads to the arising of jump terms that need to be estimated separately $[1,4]$. Instead, we opt to perform integration by parts globally using a continuous counterpart $\mathbf{g}_{h} \in \mathbf{H}(\operatorname{div}, \Omega)$ of the consistent primal gradient $\nabla u_{h} \in \mathbf{L}^{2}(\Omega)$. Since the boundary values of $\hat{z}$ and $z_{h}$ are the same, the Green formula yields

$$
\int_{\Omega}\left(\hat{z}-z_{h}\right) \nabla \cdot \mathbf{g}_{h} \mathrm{~d} \mathbf{x}+\int_{\Omega} \nabla\left(\hat{z}-z_{h}\right) \cdot \mathbf{g}_{h} \mathrm{~d} \mathbf{x}=0 .
$$

Therefore, the residual weighted by the dual error can be written as follows

$$
\begin{aligned}
\rho\left(\hat{z}-z_{h}, u_{h}\right) & =\int_{\Omega}\left(\hat{z}-z_{h}\right)\left(f-\nabla \cdot\left(\mathbf{v} u_{h}-\varepsilon \mathbf{g}_{h}\right)\right) \mathrm{d} \mathbf{x} \\
& +\varepsilon \int_{\Omega} \nabla\left(\hat{z}-z_{h}\right) \cdot\left(\mathbf{g}_{h}-\nabla u_{h}\right) \mathrm{d} \mathbf{x} .
\end{aligned}
$$


Due to the continuity of $\mathbf{g}_{h}$, there are no jump terms in this formula. Moreover, the magnitude of $f-\nabla \cdot\left(\mathbf{v} u_{h}-\varepsilon \mathbf{g}_{h}\right)$ yields a realistic estimate of the local error, whereas the consistent residual $f-\nabla \cdot\left(\mathbf{v} u_{h}-\varepsilon \nabla u_{h}\right)$ degenerates into $f-\nabla \cdot\left(\mathbf{v} u_{h}\right)$ for linear finite element approximations. A similar representation of the diffusive term can be found in $[11,12,15]$, where (i) both $\mathbf{g}_{h}$ and $\nabla \hat{z}$ are defined as averaged gradients, (ii) superconvergence is required, (iii) neither convective terms nor Galerkin orthogonality errors are taken into account.

A wealth of gradient recovery techniques are available for postprocessing and error estimation purposes $[21,22]$. For example, the averaged gradient $\mathbf{g}_{h}$ can be defined as the $L^{2}$ projection of $\nabla u_{h}$ onto a subspace $\mathbf{V}_{h}$ of $\mathbf{H}(\operatorname{div}, \Omega)$

$$
\int_{\Omega} \mathbf{w}_{h} \cdot \mathbf{g}_{h} \mathrm{~d} \mathbf{x}=\int_{\Omega} \mathbf{w}_{h} \cdot \nabla u_{h} \mathrm{~d} \mathbf{x}, \quad \forall \mathbf{w}_{h} \in \mathbf{V}_{h}
$$

Let the approximate solution $u_{h}$ and gradient $\mathbf{g}_{h}$ be interpolated using the same set of piecewise-polynomial basis functions $\left\{\varphi_{i}\right\}$. Then the algebraic systems associated with the $L^{2}$ projection (17) can be written in the form

$$
M_{C} \mathbf{g}=\mathbf{q}
$$

where the mass matrix $M_{C}=\left\{m_{i j}\right\}$ and load vector $\mathbf{q}=\left\{q_{i}\right\}$ are defined by

$$
m_{i j}=\int_{\Omega} \varphi_{i} \varphi_{j} \mathrm{~d} \mathbf{x}, \quad \mathbf{q}_{i}=\int_{\Omega} \varphi_{i} \nabla u_{h} \mathrm{~d} \mathbf{x}, \quad \forall i, j .
$$

In the case of linear or multilinear finite elements, the lumped mass matrix

$$
M_{L}=\operatorname{diag}\left\{m_{i}\right\}, \quad m_{i}=\int_{\Omega} \varphi_{i} \mathrm{~d} \mathbf{x}=\sum_{j} m_{i j}
$$

is a good approximation to $M_{C}$. For efficiency reasons, it is worthwhile to consider $\mathbf{g}=M_{L}^{-1} \mathbf{q}$ or solve system (18) by the following iterative algorithm

$$
M_{L} \mathbf{g}^{(m+1)}=\mathbf{q}+\left(M_{L}-M_{C}\right) \mathbf{g}^{(m)}, \quad m=0, \ldots, M-1 .
$$

For practical purposes, as few as $M=3$ iterations are enough. The lumpedmass version corresponds to $\mathbf{g}^{(0)}=0$ and $M=1$. The resulting $\mathbf{g}=\mathbf{g}^{(1)}$ is not as accurate as a smooth solution to (18) but devoid of undershoots and overshoots in regions where $u_{h}$ changes abruptly. The flux-corrected transport (FCT) algorithm can be used to perform adaptive mass lumping so as to achieve an accurate and nonoscillatory approximation of steep gradients $[14]$.

\section{Localization of global quantities}

The representation of $j\left(u-u_{h}\right)$ in terms of computable integrals over $\Omega$ makes it possible to verify the accuracy of the approximate solution $u_{h}$ but is of little 
help in finding the regions in which the computational mesh is too coarse or too fine. To obtain an error estimate of the form (10), it is necessary to localize global errors, i.e., distribute them among individual nodes and/or elements.

In the literature, the Cauchy-Schwarz inequality is frequently employed to derive element contributions $\eta_{k}$ to the upper bound for $\rho\left(\hat{z}-z_{h}, u_{h}\right)$. This practice is not to be recommended since it results in a strong overestimation of the global error [9] and leads to an oscillatory distribution of local errors. The latter deficiency is particularly pronounced in the 1D case if the 'exact' dual solution $\hat{z}$ is constructed from $z_{h}$ using higher-order interpolation [15].

Building on the methodology developed by Schmich and Vexler [18], we refrain from using the Cauchy-Schwarz inequality and begin with decomposition of the target functional $j\left(u-u_{h}\right)$ into nodal contributions. A straightforward definition of the local error indicators $\Phi_{i}$ and $\Psi_{i}$ for estimate (10) is

$$
\Phi_{i}=\left|z_{i} \rho\left(\psi_{i}-\varphi_{i}, u_{h}\right)\right|, \quad \Psi_{i}=\left|z_{i} \rho\left(\varphi_{i}, u_{h}\right)\right|,
$$

where the weighted residuals are evaluated by formulae (16) and (5), respectively. If the residual is orthogonal to the test function $\varphi_{i}$, then $\Psi_{i}=0$. A nonvanishing value of $\Psi_{i}$ implies that Galerkin orthogonality does not hold.

Using the fact that Lagrange basis functions sum to unity $\left(\sum_{i} \varphi_{i} \equiv 1\right)$, the share of node $i$ in the upper bound for (16) can be redefined as follows

$$
\begin{aligned}
\Phi_{i} & =\int_{\Omega} \varphi_{i}\left|\left(\hat{z}-z_{h}\right)\left(f-\nabla \cdot\left(\mathbf{v} u_{h}-\varepsilon \mathbf{g}_{h}\right)\right)\right| \mathrm{d} \mathbf{x} \\
& +\varepsilon \int_{\Omega} \varphi_{i}\left|\nabla\left(\hat{z}-z_{h}\right) \cdot\left(\mathbf{g}_{h}-\nabla u_{h}\right)\right| \mathrm{d} \mathbf{x} .
\end{aligned}
$$

The result depends not only on $z_{i}$ but also on the values of $z_{h}$ at neighboring nodes. Furthermore, no assumptions are made regarding the structure of $\hat{z}$.

By definitions (5) and (22), the Galerkin orthogonality error is measured by

$$
\Psi_{i}=\left|\int_{\Omega} z_{i}\left[\varphi_{i}\left(f-\nabla \cdot\left(\mathbf{v} u_{h}\right)\right)-\nabla \varphi_{i} \cdot\left(\varepsilon \nabla u_{h}\right)\right] \mathrm{d} \mathbf{x}\right| .
$$

To define the element contributions $\eta_{k}$, consider the continuous error function

$$
\xi(\mathbf{x})=\sum_{i} \xi_{i} \varphi_{i}(\mathbf{x}), \quad \xi_{i}=\frac{\Phi_{i}+\Psi_{i}}{\int_{\Omega} \varphi_{i} \mathrm{~d} \mathbf{x}} .
$$

Note that the denominator of $\xi_{i}$ equals the diagonal entry $m_{i}$ of the lumped mass matrix $M_{L}$ given by (20). By definition, the global error (12) equals

$$
\Phi\left(z_{h}, u_{h}\right)+\Psi\left(z_{h}, u_{h}\right)=\int_{\Omega} \xi(\mathbf{x}) \mathrm{d} \mathbf{x}=\eta\left(z_{h}, u_{h}\right)
$$


and admits the following decomposition into individual element contributions

$$
\eta\left(z_{h}, u_{h}\right)=\sum_{k} \eta_{k}, \quad \eta_{k}=\int_{\Omega_{k}} \xi(\mathbf{x}) \mathrm{d} \mathbf{x}
$$

In a practical implementation, the midpoint rule is employed to calculate $\eta_{k}$.

The sharpness of an a posteriori error estimate is frequently measured in terms of the effectivity index $I_{\text {eff }}$ defined as the ratio of estimated and true error

$$
I_{\text {eff }}=\frac{\eta\left(z_{h}, u_{h}\right)}{\left|j\left(u-u_{h}\right)\right|} .
$$

However, this definition may turn out to be misleading when the denominator is small or zero and the evaluation of integrals is subject to rounding errors. In our experience, it is worthwhile to consider the relative effectivity index

$$
I_{\text {rel }}=\left|\frac{\eta\left(z_{h}, u_{h}\right)-\left|j\left(u-u_{h}\right)\right|}{j(u)}\right|
$$

which provides another criterion for evaluating the quality of an error estimate.

\section{$6 \quad$ Numerical experiments}

A simple test problem that illustrates the utility of the above goal-oriented error estimates is the one-dimensional convection-diffusion equation

$$
\mathrm{Pe} \frac{\mathrm{d} u}{\mathrm{~d} x}-\frac{\mathrm{d}^{2} u}{\mathrm{~d} x^{2}}=0 \quad \text { in } \quad \Omega=(0,1) .
$$

The Peclet number $\mathrm{Pe}=\frac{v}{\varepsilon}$ is assumed to be constant and positive. The problem statement is completed by the Dirichlet boundary conditions

$$
u(0)=0, \quad u(1)=1 .
$$

It is easy to verify that the exact solution $u$ and its gradient $u^{\prime}$ are given by

$$
u(x)=\frac{e^{\mathrm{Pe} x}-1}{e^{\mathrm{Pe}}-1}, \quad u^{\prime}(x)=\frac{\operatorname{Pe} e^{\mathrm{Pe} x}}{e^{\mathrm{Pe}}-1} .
$$

Following Cnossen et al. [5], we define the quantity of interest as follows

$$
j\left(u-u_{h}\right)=\int_{\Omega}\left(u(x)-u_{h}(x)\right) \mathrm{d} x=\frac{e^{\mathrm{Pe}}-1-\mathrm{Pe}}{\mathrm{Pe}\left(e^{\mathrm{Pe}}-1\right)}-\int_{\Omega} u_{h}(x) \mathrm{d} x .
$$

If the numerical solution $u_{h}$ is bounded by its endpoint values 0 and 1 , as required by the discrete maximum principle (DMP) for elliptic problems, then negative values of $j\left(u-u_{h}\right)$ imply that $u_{h}$ is overly diffusive. Thus, the above target functional makes it possible to assess the amount of numerical diffusion. 
The associated dual problem (7) is endowed with the homogeneous Dirichlet boundary conditions and can also be solved analytically. The result is [5]

$$
\begin{aligned}
& z(x)=\frac{e^{\mathrm{Pe}(1-x)}+x\left(e^{\mathrm{Pe}}-1\right)-e^{\mathrm{Pe}}}{\operatorname{Pe}\left(1-e^{\mathrm{Pe}}\right)}, \\
& z^{\prime}(x)=\frac{-\mathrm{Pe} e^{\mathrm{Pe}(1-x)}+e^{\mathrm{Pe}}-1}{\operatorname{Pe}\left(1-e^{\mathrm{Pe}}\right)} .
\end{aligned}
$$

At large values of the Peclet number Pe, the primal and the dual problems are both singularly perturbed, which manifests itself in the formation of boundary layers in the neighborhood of the endpoints $x=1$ and $x=0$, respectively.

In the below numerical study, the nodal values of the approximate solutions $u_{h}$ and $z_{h}$ are computed on a uniform mesh with spacing $h=0.1$ and interpolated using ten linear finite elements. The dual solution $z$ is approximated by the quadratic interpolant $\hat{z}$ of the $N=1 / h+1$ nodal values $\left\{z_{i}\right\}$. The use of quadratic bubble functions was found to produce comparable results for this particular test problem. The computation of the averaged gradient $g \approx u^{\prime}$ is performed using the lumped-mass $L^{2}$ projection with linear elements. This approach is equivalent to approximating $u^{\prime}\left(x_{i}\right)$ by the central difference

$$
g_{i}=\frac{u_{i+1}-u_{i-1}}{2 h}, \quad i=1, \ldots, N-1
$$

at internal points and by a first-order forward/backward difference otherwise

$$
g_{0}=\frac{u_{1}-u_{0}}{h}, \quad g_{N}=\frac{u_{N}-u_{N-1}}{h} .
$$

To obtain second-order accuracy, we employ the one-sided approximations

$$
g_{0}=-\frac{3 u_{0}-4 u_{1}+u_{2}}{2 h}, \quad g_{N}=\frac{u_{N-2}-4 u_{N-1}+3 u_{N}}{2 h} .
$$

A typical discretization of equation (30) can be written in the generic form

$$
\begin{array}{r}
\mathrm{Pe} \frac{\left(1+\alpha_{i-1 / 2}\right)\left(u_{i}-u_{i-1}\right)+\left(1-\alpha_{i+1 / 2}\right)\left(u_{i+1}-u_{i}\right)}{2 h} \\
-\frac{u_{i-1}-2 u_{i}+u_{i+1}}{h^{2}}=0, \quad i=1, \ldots, N-1,
\end{array}
$$

where the diffusive term is approximated by a second-order central difference.

The approximation of the convective term represents a linear or nonlinear combination of forward $\left(\alpha_{i \pm 1 / 2}=-1\right)$ and backward $\left(\alpha_{i \pm 1 / 2}=1\right)$ differences. Due to the assumption that $\mathrm{Pe}>0$, the latter setting corresponds to the classical upwind difference scheme (UDS) which satisfies the DMP unconditionally but is only first-order accurate. The average of forward and backward differences $\left(\alpha_{i \pm 1 / 2}=0\right)$ corresponds to the central difference scheme (CDS) of second order. The same approximation is obtained with linear finite elements. Hence, numerical solutions exhibit Galerkin orthogonality 
but a violation of the discrete maximum principle and formation of spurious oscillations are possible. The CDS scheme is guaranteed to be nonoscillatory only if $h$ satisfies

$$
\text { Pe } h \leq 2 \text {. }
$$

The third discretization to be considered is a nonlinear total variation diminishing (TVD) scheme $[8,13,20]$. The corresponding correction factors $\alpha_{i+1 / 2}$ depend on the slope ratio $r_{i}$ which serves as the smoothness indicator

$$
r_{i}=\frac{u_{i+1}-u_{i}}{u_{i}-u_{i-1}}, \quad i=1, \ldots, N-1
$$

For example, the use of the monotonized centered (MC) limiter function yields

$$
\alpha_{i+1 / 2}=1-\max \left\{0, \min \left\{2, \frac{1+r_{i}}{2}, 2 r_{i}\right\}\right\}
$$

and the resulting TVD scheme (38) can be shown to possess the DMP property.

The results for $\mathrm{Pe}=10$ and $\mathrm{Pe}=100$ are shown in Figures 1-3, where the smooth curves represent the continuous functions $u, z$, and $g$. The corresponding numerical solutions are depicted as circles connected by straight lines, while the distribution of $\eta_{k}$ is displayed as a bar plot. The sums of node/element contributions and the relative effectivity index $I_{\text {rel }}$ for each scheme are listed in Tables 1-3. The discrepancy between the true and estimated errors is remarkably small as compared to the magnitude of the target functional.

The distribution of weighted element contributions reflects the qualitative behavior of local errors and indicates that stronger mesh refinement is required in the vicinity of boundary layers as the Peclet number increases. The Galerkin orthogonality error $\Psi\left(z_{h}, u_{h}\right)$ is negligible for the finite element discretization (CDS) but becomes dominant in the case of UDS and TVD solutions at large Peclet numbers. The error estimates for $\mathrm{Pe}=100$ are particularly sharp since $\Phi\left(z_{h}, u_{h}\right)$ is negligible, while $\Psi\left(z_{h}, u_{h}\right)$ is computable. These results indicate that the setting $\hat{z}=z_{h}$ is to be recommended for TVD-like schemes that violate Galerkin orthogonality only in regions of insufficient mesh resolution.

\section{Conclusions}

A posteriori error control for numerical approximations to convection-diffusion equations was addressed. The interplay between various kinds of errors that affect the quantities of interest was discussed. Goal-oriented error estimation based on the duality argument was shown to provide a proper control of numerical errors. A possible violation of Galerkin orthogonality was taken into account using a node-based approach to localization of errors. A 1D numerical study was included to illustrate the implications of upwinding and 
flux limiting in non-Galerkin approximations to convection-dominated transport problems. It turns out that the associated Galerkin orthogonality error provides a useful criterion for mesh adaptation purposes. Two-dimensional results for steady hyperbolic and elliptic problems will be presented in a forthcoming paper.

\section{Acknowledgements}

This research was supported by the German Research Association (DFG) under grants KU 1530/3-2 and KU 1530/5-1. The funding by the Academy of Finland under project No. 124619 is also gratefully acknowledged. The authors would like to thank Prof. Boris Vexler (TU München) for valuable remarks.

\section{References}

[1] W. Bangerth and R. Rannacher, Adaptive finite element methods for differential equations. Lectures in Mathematics, ETH Zürich, Birkhäuser, 2003.
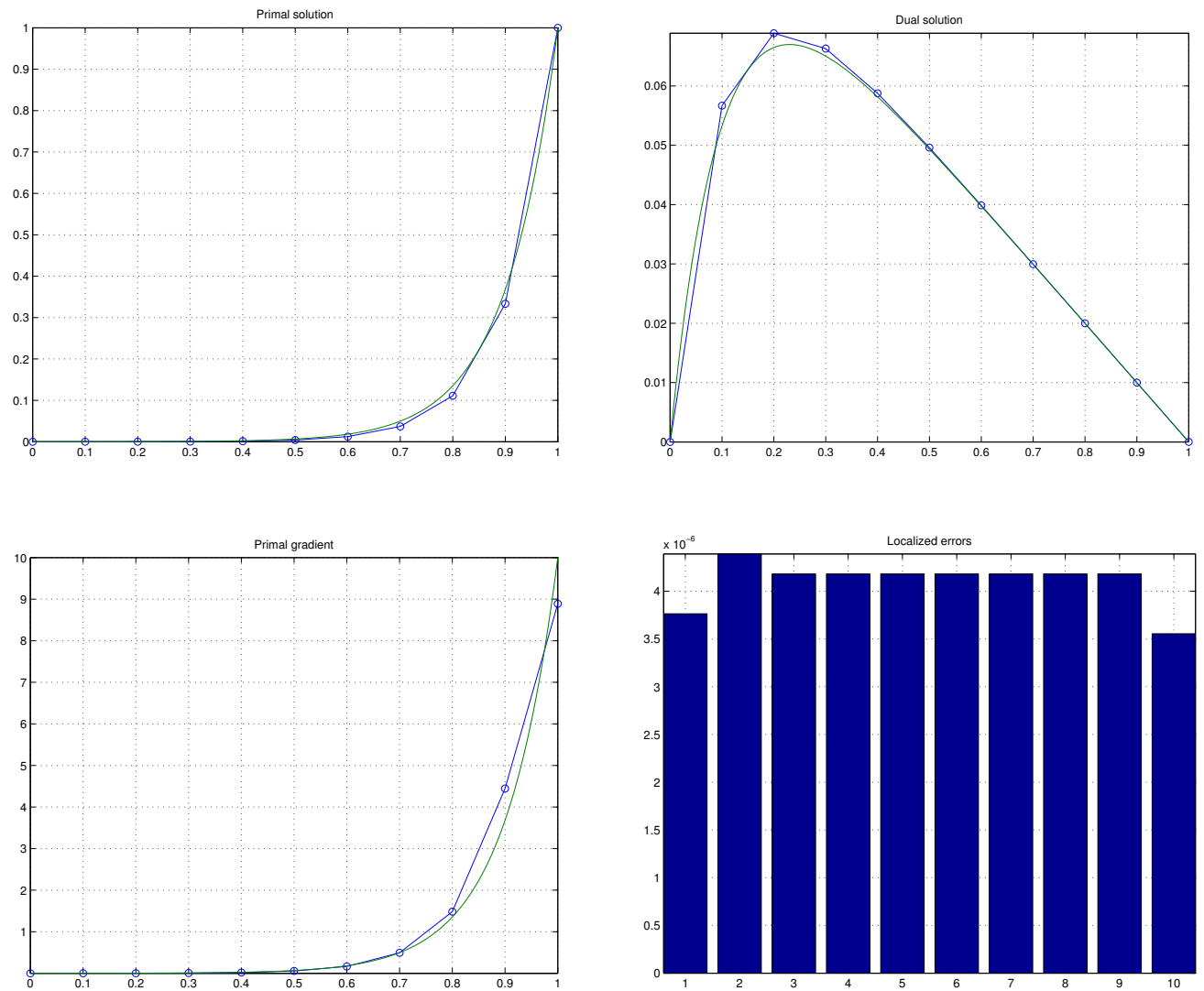

Figure 1: CDS scheme: results and error indicators $\eta_{k}$ for $\mathrm{Pe}=10$. 
[2] R. Becker, Mesh adaptation for stationary flow control, J. Math. Fluid Mech. 3 (2001) 317-341.

[3] R. Becker and R. Rannacher, A feed-back approach to error control in finite element methods: Basic analysis and examples. East-West J. Numer. Math. 4 (1996) 237-264.

[4] R. Becker and R. Rannacher, An optimal control approach to a posteriori error estimation in finite element methods. Acta Numerica 10 (2001) 1101.

[5] J.M. Cnossen, H. Bijl, M.I. Gerritsma and B. Koren, Aspects of goaloriented model-error estimation in convection-diffusion problems. Report MAS-E0703, Centrum voor Wiskunde en Informatica, Amsterdam, 2007.

[6] C. Johnson, A new paradigm for adaptive finite element methods. In: J. Whiteman (ed.), The Mathematics of Finite Elements and Applications, John Wiley \& Sons, Chichester, 1994, 105-120.

[7] C. Johnson and P. Hansbo, Adaptive finite elements in computational mechanics. Comput. Methods Appl. Mech. Engrg. 101 (1992) 143-181.
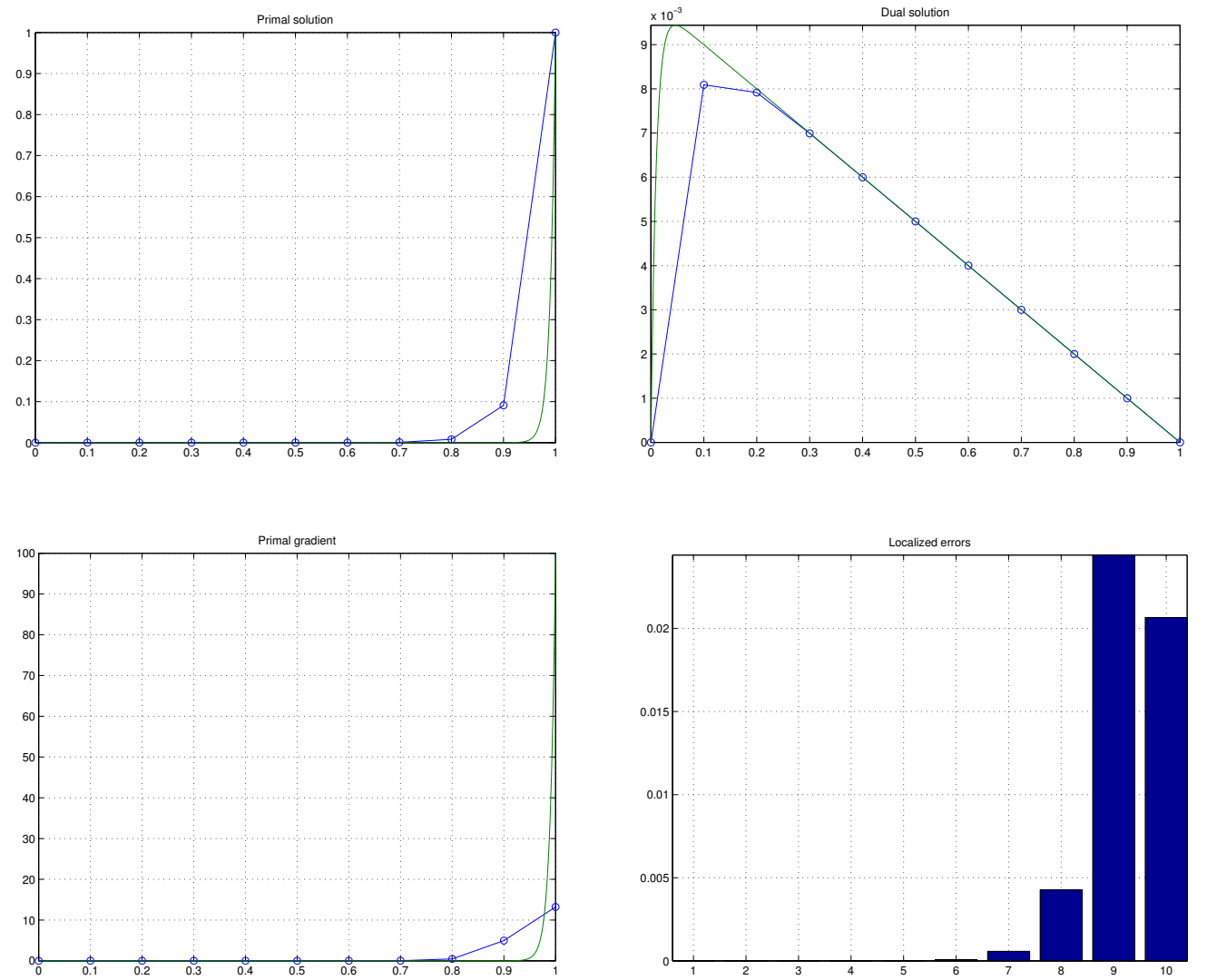

Figure 2: UDS scheme: results and error indicators $\eta_{k}$ for $\mathrm{Pe}=100$. 
[8] A. Harten, High resolution schemes for hyperbolic conservation laws. J. Comput. Phys. 49 (1983) 357-393.

[9] R. Hartmann, Adaptive FE methods for conservation equations. In: H. Freistühler and G. Warnecke (eds.), Hyperbolic Problems: Theory, Numerics, Applications, ISNM 141. Birkhäuser, Basel, 2001, 495-503.

[10] R. Hartmann and P. Houston, Adaptive discontinuous Galerkin finite element methods for nonlinear hyperbolic conservation laws. SIAM J. Sci. Comput. 24 (2002) 979-1004.

[11] S. Korotov, A posteriori error estimation of goal-oriented quantities for elliptic type BVPs. J. Comput. Appl. Math. 191 (2006) 216-227.

[12] S. Korotov, P. Neittaanmäki and S. Repin, A posteriori error estimation of goal-oriented quantities by the superconvergence patch recovery. $J$. Numer. Math. 11 (2003) 33-59.

[13] D. Kuzmin and M. Möller, Algebraic flux correction I. Scalar conservation laws. In: D. Kuzmin, R. Löhner, S. Turek (eds) Flux-Corrected Transport: Principles, Algorithms, and Applications. Springer, 2005, 155206.
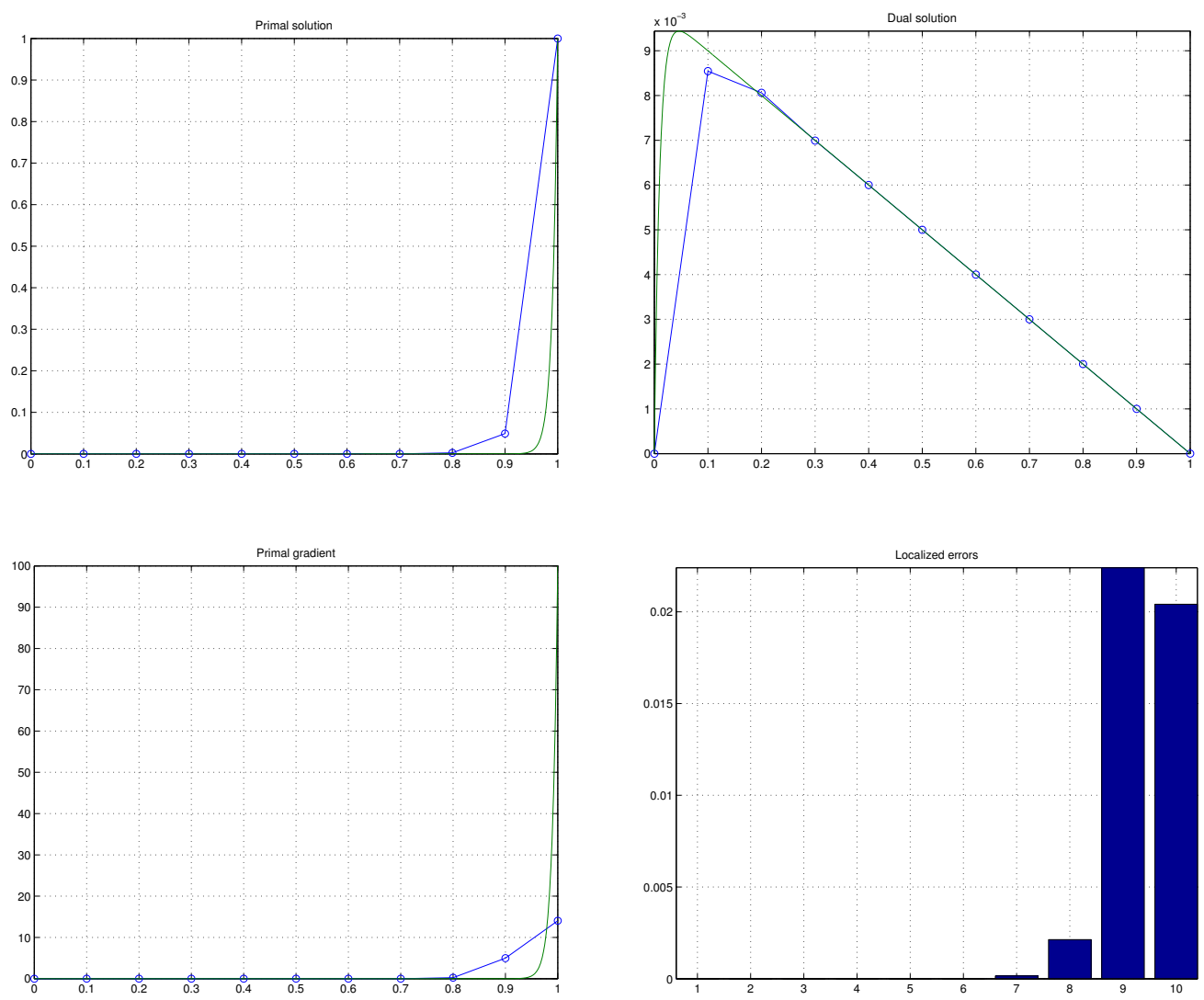

Figure 3: TVD scheme: results and error indicators $\eta_{k}$ for $\mathrm{Pe}=100$. 
Table 1: CDS scheme: exact vs. estimated global error.

\begin{tabular}{|c|c|c|c|c|c|c|}
\hline $\mathrm{Pe}$ & $|j(u)|$ & $\left|j\left(u-u_{h}\right)\right|$ & $\Phi\left(z_{h}, u_{h}\right)$ & $\Psi\left(z_{h}, u_{h}\right)$ & $\eta\left(z_{h}, u_{h}\right)$ & $I_{\text {rel }}$ \\
\hline \hline 1 & $4.18 \mathrm{e}-1$ & $7.67 \mathrm{e}-4$ & $7.80 \mathrm{e}-4$ & $4.09 \mathrm{e}-16$ & $7.80 \mathrm{e}-4$ & $3.05 \mathrm{e}-5$ \\
\hline 10 & $1.00 \mathrm{e}-1$ & $2.84 \mathrm{e}-5$ & $4.10 \mathrm{e}-5$ & $3.56 \mathrm{e}-18$ & $4.10 \mathrm{e}-5$ & $1.25 \mathrm{e}-4$ \\
\hline
\end{tabular}

Table 2: UDS scheme: exact vs. estimated global error.

\begin{tabular}{|c|c|c|c|c|c|c|}
\hline $\mathrm{Pe}$ & $|j(u)|$ & $\left|j\left(u-u_{h}\right)\right|$ & $\Phi\left(z_{h}, u_{h}\right)$ & $\Psi\left(z_{h}, u_{h}\right)$ & $\eta\left(z_{h}, u_{h}\right)$ & $I_{\text {rel }}$ \\
\hline \hline 1 & $4.18 \mathrm{e}-1$ & $4.52 \mathrm{e}-3$ & $7.38 \mathrm{e}-4$ & $3.58 \mathrm{e}-3$ & $4.32 \mathrm{e}-3$ & $4.79 \mathrm{e}-4$ \\
\hline 10 & $1.00 \mathrm{e}-1$ & $4.91 \mathrm{e}-2$ & $3.06 \mathrm{e}-4$ & $4.76 \mathrm{e}-2$ & $4.79 \mathrm{e}-2$ & $1.21 \mathrm{e}-2$ \\
\hline 100 & $1.00 \mathrm{e}-2$ & $5.00 \mathrm{e}-2$ & $1.59 \mathrm{e}-9$ & $5.00 \mathrm{e}-2$ & $5.00 \mathrm{e}-2$ & $1.21 \mathrm{e}-8$ \\
\hline
\end{tabular}

Table 3: TVD scheme: exact vs. estimated global error.

\begin{tabular}{|c|c|c|c|c|c|c|}
\hline $\mathrm{Pe}$ & $|j(u)|$ & $\left|j\left(u-u_{h}\right)\right|$ & $\Phi\left(z_{h}, u_{h}\right)$ & $\Psi\left(z_{h}, u_{h}\right)$ & $\eta\left(z_{h}, u_{h}\right)$ & $I_{\text {rel }}$ \\
\hline \hline 1 & $4.18 \mathrm{e}-1$ & $1.03 \mathrm{e}-3$ & $7.74 \mathrm{e}-4$ & $2.60 \mathrm{e}-4$ & $1.03 \mathrm{e}-3$ & $1.34 \mathrm{e}-5$ \\
\hline 10 & $1.00 \mathrm{e}-1$ & $1.51 \mathrm{e}-2$ & $9.12 \mathrm{e}-5$ & $1.50 \mathrm{e}-2$ & $1.51 \mathrm{e}-2$ & $3.81 \mathrm{e}-5$ \\
\hline 100 & $1.00 \mathrm{e}-2$ & $4.51 \mathrm{e}-2$ & $4.23 \mathrm{e}-9$ & $4.51 \mathrm{e}-2$ & $4.51 \mathrm{e}-2$ & $1.97 \mathrm{e}-7$ \\
\hline
\end{tabular}

[14] R. Löhner, Finite element formulations and advanced applications. In: H.G. Matthies and R. Ohayon (eds), ECCOMAS course on Advanced Computational Methods for Fluid-Structure Interaction, 3-7 May 2006, Ibiza, Spain.

[15] J. S. Ovall, Asymptotically exact functional error estimators based on superconvergent gradient recovery. Numer. Math. 102:3 (2006) 543-558.

[16] A. M. Sauer-Budge and J. Peraire, Computing bounds for linear functionals of exact weak solutions to the advection-diffusion-reaction equation. SIAM J. Sci. Comput. 26 (2004) 636-652.

[17] S. Prudhomme and J. T. Oden, Computable error estimators and adaptive techniques for fluid flow problems. In: T. J. Barth and H. Deconinck (eds), Error Estimation and Adaptive Discretization Methods in Computational Fluid Dynamics, Lect. Notes Comput. Sci. Eng. 25, Springer, Berlin, 2003, 207-268.

[18] M. Schmich and B. Vexler, Adaptivity with dynamic meshes for spacetime finite element discretizations of parabolic equations. SIAM J. Sci. Comput. 30:1 (2008) 369-393. 
[19] P. Šolin and L. Demkowicz, Goal-oriented $h p$-adaptivity for elliptic problems. Comput. Methods Appl. Mech. Engrg. 193 (2004) 449-468.

[20] P.K. Sweby, High resolution schemes using flux limiters for hyperbolic conservation laws. SIAM J. Numer. Anal. 21 (1984) 995-1011.

[21] O.C. Zienkiewicz and J.Z. Zhu, The superconvergent patch recovery and a posteriori error estimates. Part 1: The recovery techniques. Int. J. Numer. Methods Engrg. 33 (1992) 1331-1364.

[22] O.C. Zienkiewicz and J.Z. Zhu, The superconvergent patch recovery and a posteriori error estimates. Part 2: Error estimates and adaptivity. Int. J. Numer. Methods Engrg. 33 (1992) 1365-1382. 
(continued from the back cover)

A556 Lourenço Beirão da Veiga, Jarkko Niiranen, Rolf Stenberg

A posteriori error analysis for the Morley plate element with general boundary conditions

December 2008

A555 Juho Könnö, Rolf Stenberg

Finite element analysis of composite plates with an application to the paper cockling problem

December 2008

A554 Lasse Leskelä

Stochastic relations of random variables and processes

October 2008

A553 Rolf Stenberg

A nonstandard mixed finite element family

September 2008

A552 Janos Karatson, Sergey Korotov

A discrete maximum principle in Hilbert space with applications to nonlinear cooperative elliptic systems

August 2008

A551 István Faragó, Janos Karatson, Sergey Korotov

Discrete maximum principles for the FEM solution of some nonlinear parabolic problems

August 2008

A550 István Faragó, Róbert Horváth, Sergey Korotov Discrete maximum principles for FE solutions of nonstationary diffusion-reaction problems with mixed boundary conditions August 2008

A549 Antti Hannukainen, Sergey Korotov, Tomás Vejchodský On weakening conditions for discrete maximum principles for linear finite element schemes

August 2008

A548 Kalle Mikkola

Weakly coprime factorization, continuous-time systems, and strong- $H^{p}$ and Nevanlinna fractions

August 2008 
HELSINKI UNIVERSITY OF TECHNOLOGY INSTITUTE OF MATHEMATICS RESEARCH REPORTS

The reports are available at $h t t p: / / m a t h . t k k . f i / r e p o r t s /$.

The list of reports is continued inside the back cover.

A562 Antti H. Niemi

A bilinear shell element based on a refined shallow shell model

December 2008

A561 Antti Hannukainen, Sergey Korotov, Michal Krizek

On nodal superconvergence in 3D by averaging piecewise linear, bilinear, and trilinear FE approximations

December 2008

A560 Sampsa Pursiainen

Computational methods in electromagnetic biomedical inverse problems

November 2008

A559 Sergey Korotov, Michal Krizek, Jakub Solc

On a discrete maximum principle for linear FE solutions of elliptic problems with a nondiagonal coefficient matrix

November 2008

A558 José Igor Morlanes, Antti Rasila, Tommi Sottinen

Empirical evidence on arbitrage by changing the stock exchange

December 2008

ISBN 978-951-22-9758-0 (print)

ISBN 978-951-22-9759-7 (PDF)

ISSN 0784-3143 (print)

ISSN 1797-5867 (PDF) 